\title{
Endocarditis infecciosa fatal por Corynebacterium pseudodiphtheriticum
}

\author{
Santiago Pérez-Parra, Alejandro Peña, Enrique Blanca y Federico García
}

\section{Letal endocarditis due to Corynebacterium pseudodiphtheriticum}

We present a case report of a infective endocarditis by Corynebacterium pseudodiphtheriticum in a young patient eight years old with bicuspid aortic valve that led to a severe neurological embolism and death. Corynebacterium pseudodiphtheriticum is part of the nasopharyngeal and skin flora. However, there are cases reported of endocarditis usually associated with immunocompromised patients that mostly presented a poor clinical outcome.

Key words: Corynebacterium pseudodiphteriticum, endocarditis, cerebral embolism.

Palabras clave: Corynebacterium pseudodiphteriticum, endocarditis, embolia cerebral.

\section{Introducción}

$\mathrm{E}$ 1 género Corynebacterium agrupa a un grupo diverso de bacilos grampositivos pleomórficos, catalasa positivos, no esporulados. Tiene una distribución ubicua en la naturaleza, y forma parte de la microbiota de la piel humana ${ }^{1}$. Corynebacterium pseudodiphtheriticum es un comensal de la orofaringe ${ }^{2,3}$; sin embargo, puede causar infecciones respiratorias (traqueítis, neumonía o bronquitis en pacientes en ventilación mecánica), generalmente en pacientes inmunocomprometidos ${ }^{4}$. El aislamiento de C. pseudodiphtheriticum como agente responsable de endocarditis ocurre aproximadamente en $14 \%$ de todos los casos descritos de endocarditis por el género Corynebacterium, siendo menos frecuente que otras especies, y casi siempre se relaciona a un mal pronóstico clínico ${ }^{5,6}$.

\section{Caso clínico}

Varón de 8 años, sin antecedentes clínicos de interés, que consultó al servicio de urgencia por un cuadro clínico de fiebre hasta $39,5^{\circ} \mathrm{C}$ de 14 días de evolución, asociada a escalofríos, palidez de piel, adenopatías cervicales bilaterales, decaimiento e inapetencia. Había sido tratado con azitromicina $500 \mathrm{mg}$ al día, por cinco días. En la exploración física, destacaba la presencia de un soplo sistólico grado II/III en el foco auscultatorio correspondiente al ventrículo izquierdo. El electroencefalograma y la radiografía de tórax, no evidenciaron hallazgos patológicos. Los exámenes de laboratorio mostraron la presencia de marcadores inflamatorios: leucocitos de 10.500 céls $/ \mathrm{ml}$, una neutrofilia de $70 \%$ y una PCR elevada $(119 \mathrm{mg} / \mathrm{L})$. Se inició tratamiento con cefotaxima i.v. $200 \mathrm{mg} / \mathrm{kg} /$ día previa toma de hemocultivos seriados en dos días consecutivos. A las $24 \mathrm{~h}$ de incubación, ambos hemocultivos fueron positivos. En la tinción de Gram se observaron bacilos grampositivos pleomórficos en forma de "V". La identificación directa del microorganismo a partir de los hemocultivos positivos mediante espectrometría de masas MALDI-TOF fue C. pseudodiphtheriticum. En este contexto, se realizó una ecocardiografía en la que se observó la existencia de una formación vegetativa de $10 \mathrm{~mm}$ (Figura 1) del lado ventricular izquierdo, provocando insuficiencia moderada, sin repercusión en la hemodinamia y función cardíaca. Asimismo, se constató la presencia de una valvulopatía aórtica bicúspide. Con el diagnóstico de una endocarditis infecciosa se agregó gentamicina ( $6 \mathrm{mg} / \mathrm{kg} / \mathrm{día})$ a la terapia a la espera de la identificación definitiva del microorganismo y su perfil de susceptibilidad antimicrobiana. A las $24 \mathrm{~h}$ de incubación en medios de cultivo sólidos se observó el crecimiento de colonias grises en placas de agar sangre, catalasa positivas. La identificación mediante secuenciación del gen ARN 16S confirmó el aislamiento de C. pseudodiphtheriticum. El antibiograma con epsilometría (E-test) mostró resistencia a azitromicina (CIM $>256 \mu \mathrm{g} / \mathrm{ml})$ y sensibilidad (CIM) a amoxicilina/ác. clavulánico $(0,016$ $\mu \mathrm{g} / \mathrm{ml})$, cefotaxima $(0,38 \mu \mathrm{g} / \mathrm{ml})$, ciprofloxacina $(0,5 \mu \mathrm{g} /$ $\mathrm{ml})$, linezolid $(0,016 \mu \mathrm{g} / \mathrm{ml})$, penicilina $\mathrm{G}(0,25 \mu \mathrm{g} / \mathrm{ml}) \mathrm{y}$ vancomicina $(0,38 \mu \mathrm{g} / \mathrm{ml})$.

El paciente presentó una evolución clínica favorable, remitiendo su cuadro febril antes de las $48 \mathrm{~h}$ de iniciada la terapia antimicrobiana combinada. Dada su buena evolución, habiendo negativizado los hemocultivos y sin cambios imagenológicos no se planteó una cirugía cardíaca en ese momento, salvo que hubiera algún deterioro clínico.

A los 16 días de internación, el paciente presentó
Complejo Hospitalario Universitario de Granada, Hospital San Cecilio-PTS. Instituto de Investigación Biosanitaria IBS. Granada, España.

Servicio de Microbiología (SPP $A P, F G)$.

Servicio de Cardiología infantil (EB).

Recibido: 22 de septiembre de 2015

Aceptado: 26 de enero de 2016

Correspondencia a:

Santiago Pérez-Parra

santperez85@gmail.com 
Figura 1. Ecocardiografía transtorácica que muestra la presencia de una masa excrecente de $1 \mathrm{~cm}$ que ocupa la mayor parte del tracto de salida aórtico a nivel de la cara ventricular.

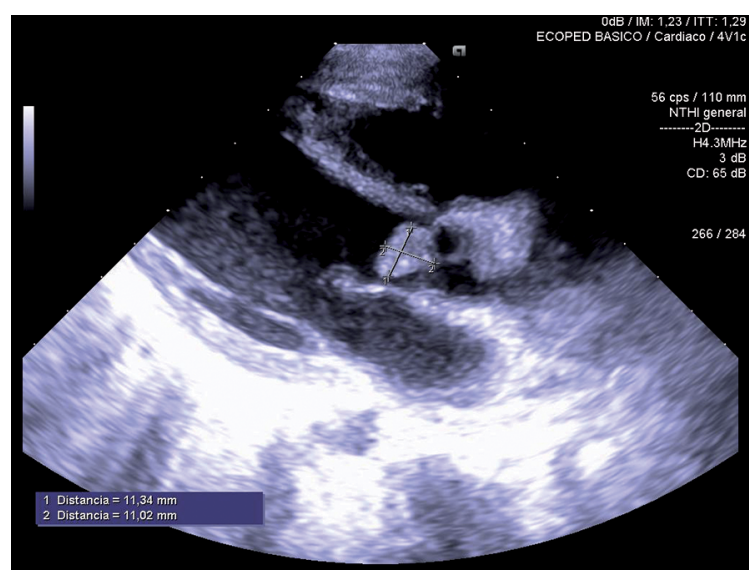

y Cauda y cols. ${ }^{11}$, el uso de un glucopéptido asociado a gentamicina. Además, este microorganismo es habitualmente sensible a ciprofloxacina y tetraciclinas, siendo a menudo resistente a macrólidos, como eritromicina o azitromicina ${ }^{4,10,12}$.

Hasta ahora, poco se sabe sobre los factores de virulencia y patogenia de este microorganismo. Roy y cols. ${ }^{13}$, describen una mayor activación del sistema inmune, con un aumento de la expresión de los receptores Tolllike, e interleuquinas (IL-6 y IL-1 $\beta$ ) de esta bacteria en células epiteliales corneales en seres humanos. En este contexto, sugieren que este incremento en la activación del sistema inmune podría producirse en otros tejidos en personas sanas para mantener en un estilo de vida comensal a una bacteria potencialmente patógena. Souza y cols. ${ }^{14}$, describieron recientemente la capacidad de $C$. pseudodiphtheriticum de formar biopelículas en modelos in-vitro, por lo que el éxito terapéutico en estos casos, estaría condicionado directamente a la duración y la dosis del antimicrobiano, siendo de gran importancia, un tratamiento antibacteriano adecuado, prolongado y con concentraciones elevadas en sangre.

A nuestro juicio, creemos que la mala evolución ocurrida en nuestro paciente fue consecuencia en gran medida del diagnóstico tardío de la endocarditis infecciosa. La resistencia de este microorganismo a los macrólidos y el tratamiento antimicrobiano inadecuado utilizado durante las semanas previas al ingreso, pudieron haber sido determinantes en su desenlace. En el caso clínico presentado, que sucedió sobre un factor predisponente como es el bicuspidismo aórtico, y dada la buena respuesta al tratamiento antimicrobiano en un comienzo, se decidió una actitud conservadora. Asimismo, el paciente presentaba una insuficiencia cardíaca moderada, sin repercusión en la hemodinamia y en su función cardíaca por lo que no se planteó la opción quirúrgica desde un principio, salvo ante una posible no mejora evolutiva. En este contexto, el tamaño de la formación vegetativa podría constituir una indicación relativa de tratamiento quirúrgico si se le suman factores de mal pronóstico como; fallo cardíaco congestivo, fenómenos embólicos o disfunción valvular progresiva. Las opciones de tratamiento quirúrgico incluyen la reparación o sustitución valvular $^{15,16}$. Nuestro paciente presentó una embolia cerebral por lo que fue necesario sustituir la válvula cardíaca afectada, aunque la evolución posterior no fue la esperada.

El paciente presentado es un caso atípico de endocarditis infecciosa causada por C. pseudodiphtheriticum en un niño sin un cuadro infeccioso previo claro. El aislamiento de este microorganismo en la vía respiratoria, como agente causante de faringitis pseudomembranosa ${ }^{2}$ o de neumonía ${ }^{17}$ en personas sanas sin antecedentes quirúrgicos, podría significar un foco de origen o puerta de 
entrada a una bacteriemia determinando así una relación causal con endocarditis infecciosa. Aunque se necesitan más estudios para conocer los factores de virulencia, mecanismos de patogenicidad y el modo de transmisión de C. pseudodiphtheriticum, debe destacarse su capacidad patógena.

Por último, recomendamos el uso de métodos microbiológicos avanzados, como la tecnología MALDI-TOF para una identificación rápida de microorganismos en infecciones graves.

\section{Resumen}

Se presenta el caso clínico de una endocarditis infecciosa por Corynebacterium pseudodiphtheriticum en un niño de 8 años, portador de una valvulopatía aórtica bicúspide, que falleció por una embolia cerebral grave. Corynebacterium pseudodiphtheriticum forma parte de la microbiota de la piel y nasofaringe. Sin embargo, se han descrito casos de endocarditis en pacientes inmunocomprometidos, asociados, en su mayoría, a una mala evolución clínica.

\section{Referencias bibliográficas}

1.- Bernard K. The genus Corynebacterium and other medically relevant coryneformlike bacteria. J Clin Microbiol 2012; 50 3152-8.

2.- Indumathi V A, Shikha R, Suryaprakash D R. Diphtheria-like illness in a fully immunised child caused by Corynebacterium pseudodiphtheriticum. Indian J Med Microbiol 2014; 32: 443-5.

3.- Muliukin A L, Suzina N E, Mel'nikov V G, Gal'chenko V F. Dormant state and phenotypic variability of Staphylococcus aureus and Corynebacterium pseudodiphtheriticum. Mikrobiologiia 2014; 83: 15-27.

4.- Mensa J, Gatell J M, García J E, Letang E, López E, Marco F. Guía de terapéutica antimicrobiana. Barcelona: Antares; 2012.

5.- Belmares J, Detterline S, Pak J B, Parada J P. Corynebacterium endocarditis species-specific risk factors and outcomes. BMC Infect Dis 2007; 7: 4 .

6.- Johnson J N, Miller S G, Lodge A J. Corynebacterium endocarditis of a percutaneously placed transcatheter pulmonary valve. Cardiol Young 2014; 24: 932-4.

7.- Karchmer A W. Antimicrobial therapy of prosthetic valve endocarditis. Up to Date 2015 (acceso 15 de agosto de 2015).

Disponible en: http://www.uptodate.com/ contents/antimicrobial-therapy-of-prostheticvalve-endocarditis

8.- Manzella J P, Kellogg J A, Parsey K S. Corynebacterium pseudodiphtheriticum: a respiratory tract pathogen in adults. Clin Infect Dis 1995; 20: 37: 40.

9.- Ahmed K, Kawakami K, Watanabe K, Mitsushima H, Nagatake T, Matsumoto K. Corynebacterium pseudodiphtheriticum: a respiratory tract pathogen. Clin Infect Dis 1995; 20: 41-6.

10.- Morris A, Guild I. Endocarditis due to Corynebacterium pseudodiphtheriticum: five case reports, review, and antibiotic susceptibilities of nine strains. Rev Infect Dis 1991; 13: 887-92.

11.- Cauda R, Tamburrini E, Ventura G, Ortona L. Effective vancomycin therapy for Corynebacterium pseudodiphtheriticum endocarditis. South Med J 1987; 80: 1598.

12.- Soriano F, Zapardiel J, Nieto E. Antimicrobial susceptibilities of Corynebacterium species and other non-spore-forming gram-positive bacilli to 18 antimicrobial agents. Antimicrob Agents Chemother 1995; 39: 208-14.
13.- Roy S, Marla S, Praneetha D C. Recognition of Corynebacterium pseudodiphtheriticum by Tolllike receptors and up-regulation of antimicrobial peptides in human corneal epithelial cells. Virulence 2015; 6: 716-21.

14.- Souza M C, dos Santos L S, Sousa L P, Faria Y V, Ramos J N, Sabbadini P S, et al. Biofilm formation and fibrinogen and fibronectin binding activities by Corynebacterium pseudodiphtheriticum invasive strains. Antonie Van Leeuwenhoek 2015; 107: 1387-99.

15.- Baltimore R S, Gewitz M, Baddour L M, Beerman L B, Jackson M A, Lockhart P B, et al. Infective endocarditis in childhood: 2015 update: a scientific statement from the American Heart Association. Circulation 2015; 132: $1487-515$

16.- Habib G, Lancellotti P, Antunes M J, Bongiorni M G, Casalta J P, Del Zotti F. 2015 ESC Guidelines for the management of infective endocarditis. Eur Heart J 2015; 36: 3075-128.

17.- Van Roeden S E, Thijsen S F, Sankatsing S C, Limonard G J. Clinical relevance of Corynebacterium pseudodiphtheriticum in lower respiratory tract specimens. Infect Dis (Lond) 2015; 47: 862-8 . 